\title{
Approximation and Fast Calculation of Non-local Boundary Conditions for the Time-dependent Schrödinger Equation
}

Anton Arnold ${ }^{1}$, Matthias Ehrhardt ${ }^{2}$, and Ivan Sofronov ${ }^{3}$

${ }^{1}$ Universität Münster, Institut für Numerische Mathematik, Einsteinstr. 62, D-48149 Münster, Germany (http://www.math.uni-muenster.de/u/arnold/)

2 Technische Universität Berlin, Institut für Mathematik, Str. des 17. Juni 136, D-10623 Berlin, Germany (http://www.math.tu-berlin.de/ Nehrhardt/)

${ }^{3}$ Keldysh Institute of Applied Mathematics, Russian Academy of Sciences, Moscow, Russia (sofronov@spp.keldysh.ru)

Summary. We present a way to efficiently treat the well-known transparent boundary conditions for the Schrödinger equation. Our approach is based on two ideas: firstly, to derive a discrete transparent boundary condition (DTBC) based on the Crank-Nicolson finite difference scheme for the governing equation. And, secondly, to approximate the discrete convolution kernel of DTBC by sum-of-exponentials for a rapid recursive calculation of the convolution. We illustrate the efficiency of the proposed method on several examples.

A much more detailed version of this article can be found in Arnold et al. [2003].

\section{Introduction}

Discrete transparent boundary conditions for the discrete 1D-Schrödinger equation

$$
-i R\left(\psi_{j, n+1}-\psi_{j, n}\right)=\Delta^{2}\left(\psi_{j, n+1}+\psi_{j, n}\right)-w V_{j, n+\frac{1}{2}}\left(\psi_{j, n+1}+\psi_{j, n}\right),
$$

where $\Delta^{2} \psi_{j}=\psi_{j+1}-2 \psi_{j}+\psi_{j-1}, R=4 \Delta x^{2} / \Delta t, w=2 \Delta x^{2}, V_{j, n+\frac{1}{2}}:=$ $V\left(x_{j}, t_{n+\frac{1}{2}}\right), x_{j}=j \Delta x, j \in \mathbb{Z}$; and $V(x, t)=V_{-}=$const. for $x \leq 0 ; V(x, t)=$ $V_{+}=$const. for $x \geq X, t \geq 0, \psi(x, 0)=\psi^{I}(x)$, with supp $\psi^{I} \subset[0, X]$, were introduced in Arnold [1998]. The DTBC at e.g. the left boundary point $j=0$ reads, cf. Thm. 3.8 in Ehrhardt and Arnold [2001]:

$$
\psi_{1, n}-s_{0} \psi_{0, n}=\sum_{k=1}^{n-1} s_{n-k} \psi_{0, k}-\psi_{1, n-1}, \quad n \geq 1 .
$$

The convolution kernel $\left\{s_{n}\right\}$ can be obtained by explicitly calculating the inverse $Z$-transform of the function $\hat{s}(z):=\frac{z+1}{z} \hat{\ell}_{0}(z)$, where $\hat{\ell}_{0}(z)=1-i \zeta \pm$ $\sqrt{-\zeta(\zeta+2 i)}, \zeta=\frac{R}{2} \frac{z-1}{z+1}+i \Delta x^{2} V_{-}\left(\right.$choose sign such that $\left.\left|\hat{\ell}_{0}(z)\right|>1\right)$. 
Using (2) in numerical simulations permits to avoid any boundary reflections and it renders the fully discrete scheme unconditionally stable, like the Crank-Nicolson scheme (1) for the whole-space problem. However, the numerical effort to evaluate the DTBC increases linearly in $t$ and it can sharply raise the total computational costs. A strategy to overcome this drawback is the key issue of this paper.

\section{Approximation by Sums of Exponentials}

The convolution coefficients $s_{n}$ appearing in the DTBC (2) can either be obtained from (lengthy) explicit formulas or evaluated numerically: $s_{n} \approx$ $\rho^{n} N^{-1} \sum_{k=0}^{N-1} \hat{s}\left(\rho e^{i \varphi_{k}}\right) e^{i n \varphi_{k}}, n=0,1, \ldots, N-1$. Here $\varphi_{k}=2 \pi k / N$, and $\rho>1$ is a regularization parameter. For the question of choosing $\rho$ we refer the reader to Arnold et al. [2003] and the references therein.

Our fast method to calculate the discrete convolution in (2) is based on approximating these coefficients $s_{n}$ by the following ansatz (sum of exponentials):

$$
s_{n} \approx \tilde{s}_{n}:= \begin{cases}s_{n}, & n=0, \ldots, \nu-1, \\ \sum_{l=1}^{L} b_{l} q_{l}^{-n}, & n=\nu, \nu+1, \ldots,\end{cases}
$$

where $L, \nu \in \mathbb{N}$ are fixed numbers. In order to find the appropriate constants $\left\{b_{l}, q_{l}\right\}$, we fix $L$ and $\nu$ in (3) (e.g. $\left.\nu=2\right)$, and consider the Padé approximation $\frac{P_{L-1}(x)}{Q_{L}(x)}$ for the formal power series: $f(x):=s_{\nu}+s_{\nu+1} x+s_{\nu+2} x^{2}+\ldots$, $|x| \leq 1$

Theorem 1. Let the polynomial $Q_{L}(x)$ have $L$ simple roots $q_{l}$ with $\left|q_{l}\right|>1$, $l=1, \ldots, L$. Then

$$
\tilde{s}_{n}=\sum_{l=1}^{L} b_{l} q_{l}^{-n}, \quad n=\nu, \nu+1, \ldots,
$$

where

$$
b_{l}:=-\frac{P_{L-1}\left(q_{l}\right)}{Q_{L}^{\prime}\left(q_{l}\right)} q_{l}^{\nu-1} \neq 0, \quad l=1, \ldots, L .
$$

Remark 1. All our practical calculations confirm that the assumption of Theorem 1 holds for any desired $L$, although we cannot prove this.

Remark 2. According to the definition of the Padé algorithm the first $2 L+\nu-1$ coefficients are reproduced exactly: $\tilde{s}_{n}=s_{n}$ for $n=\nu, \nu+1, \ldots, 2 L+\nu-1$. For the remaining $\tilde{s}_{n}$ with $n>2 L+\nu-1$, the following estimate holds: $\left|s_{n}-\tilde{s}_{n}\right|=\mathcal{O}\left(n^{-\frac{3}{2}}\right)$. A typical graph of $\left|s_{n}\right|$ and $\left|s_{n}-\tilde{s}_{n}\right|$ versus $n$ for $L=20$ is shown in Fig. 1 (note the different scaling for both graphs). 


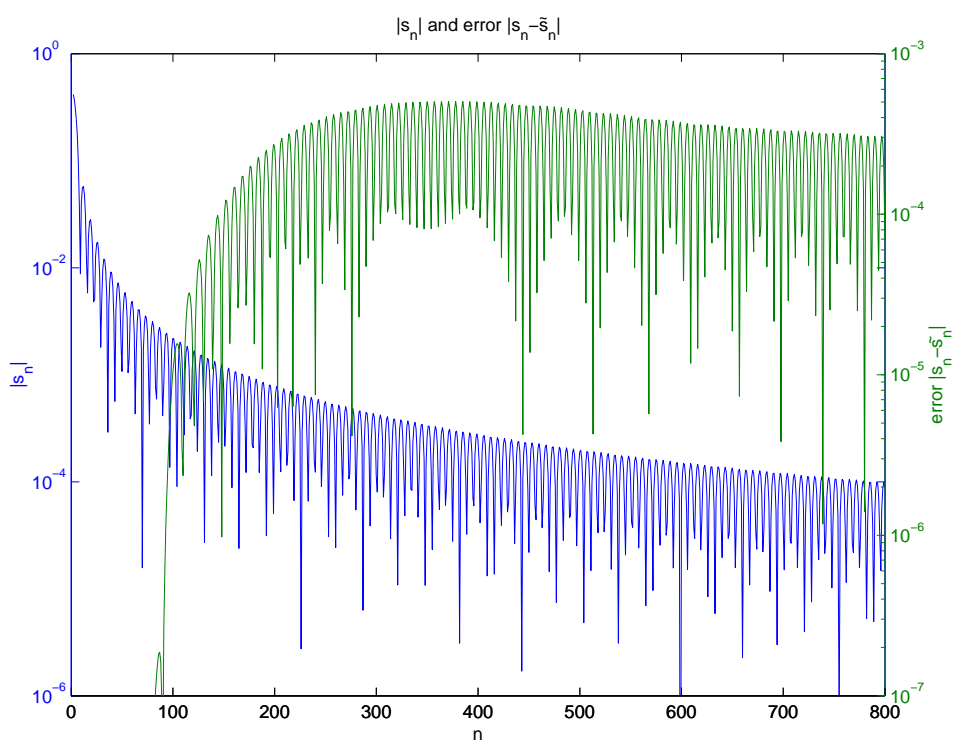

Fig. 1. Convolution coefficients $s_{n}$ (left axis, dashed line) and error $\left|s_{n}-\tilde{s}_{n}\right|$ of the convolution coefficients (right axis); $\Delta x=1 / 160, \Delta t=2 \cdot 10^{-5}, V \equiv 0(L=20)$.

\section{The Transformation Rule}

A nice property of the considered approach consists of the following: once the approximate convolution coefficients $\left\{\tilde{s}_{n}\right\}$ are calculated for particular discretization parameters $\{\Delta x, \Delta t, V\}$, it is easy to transform them into appropriate coefficients for any other discretization. We shall confine this discussion to the case $\nu=2$ :

Transformation rule 3.1 For $\nu=2$, let the rational function

$$
\hat{\tilde{s}}(z)=s_{0}+\frac{s_{1}}{z}+\sum_{l=1}^{L} \frac{b_{l}}{q_{l} z-1} \frac{1}{q_{l} z}
$$

be the $Z$-transform of the convolution kernel $\left\{\tilde{s}_{n}\right\}_{n=0}^{\infty}$ from (3), where $\left\{\tilde{s}_{n}\right\}$ is assumed to be an approximation to a DTBC for the equation (1) with a given set $\{\Delta x, \Delta t, V\}$.

Then, for another set $\left\{\Delta x_{\star}, \Delta t_{\star}, V_{\star}\right\}$, one can take the approximation

$$
\hat{\tilde{s}}^{\star}(z):=s_{0}^{\star}+\frac{s_{1}^{\star}}{z}+\sum_{l=1}^{L} \frac{b_{l}^{\star}}{q_{l}^{\star} z-1} \frac{1}{q_{l}^{\star} z},
$$

where

$$
q_{l}^{\star}:=\frac{q_{l} \bar{a}-\bar{b}}{a-q_{l} b}, \quad b_{l}^{\star}:=b_{l} q_{l} \frac{a \bar{a}-b \bar{b}}{\left(a-q_{l} b\right)\left(q_{l} \bar{a}-\bar{b}\right)} \frac{1+q_{l}^{\star}}{1+q_{l}}
$$




$$
\begin{aligned}
& a:=2 \frac{\Delta x^{2}}{\Delta t}+2 \frac{\Delta x_{\star}^{2}}{\Delta t_{\star}}+i\left(\Delta x^{2} V-\Delta x_{\star}^{2} V_{\star}\right), \\
& b:=2 \frac{\Delta x^{2}}{\Delta t}-2 \frac{\Delta x_{\star}^{2}}{\Delta t_{\star}}-i\left(\Delta x^{2} V-\Delta x_{\star}^{2} V_{\star}\right) .
\end{aligned}
$$

$s_{0}^{\star}, s_{1}^{\star}$ are the exact convolution coefficients for the parameters $\left\{\Delta x_{\star}, \Delta t_{\star}, V_{\star}\right\}$.

While the Padé-algorithm provides a method to calculate approximate convolution coefficients $\tilde{s}_{n}$ for fixed parameters $\{\Delta x, \Delta t, V\}$, the Transformation rule yields the natural link between different parameter sets $\left\{\Delta x_{\star}, \Delta t_{\star}\right.$, $\left.V_{\star}\right\}$ (and $L$ fixed).

Example 1. For $L=10$ we calculated the coefficients $\left\{b_{l}, q_{l}\right\}$ with the parameters $\Delta x=1, \Delta t=1, V=0$ and then used the Transformation 3.1 to calculate the coefficients $\left\{b_{l}^{*}, q_{l}^{*}\right\}$ for the parameters $\Delta x_{*}=1 / 160, \Delta t_{*}=2 \cdot 10^{-5}, V_{*}=$ 4500. Fig. 2 shows that the resulting convolution coefficients $\tilde{s}_{n}^{*}$ are in this example even better approximations to the exact coefficients $s_{n}$ than the coefficients $\tilde{s}_{n}$, which are obtained directly from the Padé algorithm discussed in Theorem 1. Hence, the numerical solution of the corresponding Schrödinger equation is also more accurate (cf. Fig. 5).

The Maple code that was used to calculate the coefficients $q_{l}, b_{l}$ in the approximation (3) including the explicit formulas in Transformation rule 3.1 can be downloaded from the authors' homepages.

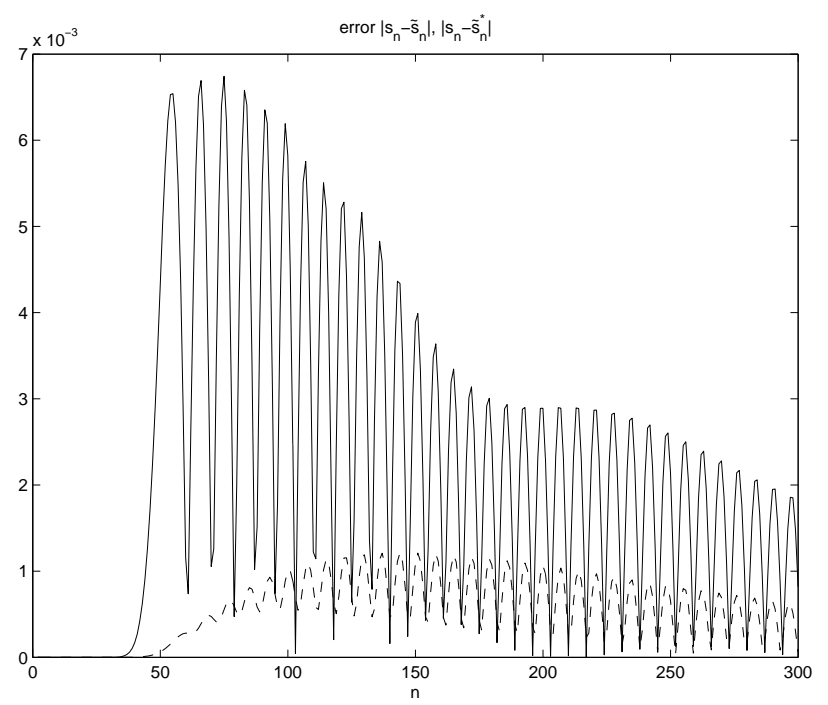

Fig. 2. Approximation error of the approximate convolution coefficients for $\nu=2$, $\Delta x=1 / 160, \Delta t=2 \cdot 10^{-5}, V=4500$ : The error of $\tilde{s}_{n}^{*}(--$-) obtained from the transformation rule and the error of $\tilde{s}_{n}(-)$ obtained from a direct Padé approximation of the exact coefficients $s_{n}$. 


\section{Fast Evaluation of the Discrete Convolution}

Given the approximation (3) of the discrete convolution kernel appearing in the DTBC (2), the convolution

$$
C^{(n)}(u):=\sum_{k=1}^{n-\nu} u_{k} \tilde{s}_{n-k}, \quad \tilde{s}_{n}=\sum_{l=1}^{L} b_{l} q_{l}^{-n}, \quad\left|q_{l}\right|>1,
$$

of a discrete function $u_{k}, k=1,2, \ldots$, can be calculated efficiently by recurrence formulas, cf. Sofronov [1998]:

Theorem 2. The function $C^{(n)}(u)$ from (11) for $n \geq \nu+1$ is represented by

$$
C^{(n)}(u)=\sum_{l=1}^{L} C_{l}^{(n)}(u)
$$

where

$$
C_{l}^{(n)}(u)=q_{l}^{-1} C_{l}^{(n-1)}(u)+b_{l} q_{l}^{-\nu} u_{n-\nu} \quad \text { for } n \geq \nu+1, \quad C_{l}^{(\nu)}(u) \equiv 0 .
$$

This recursion drastically reduces the computational effort of evaluating DTBCs for long-time computations $(n \gg 1): \mathcal{O}(L * n)$ instead of $\mathcal{O}\left(n^{2}\right)$ arithmetic operations.

\section{Numerical Examples}

In this section we shall present two examples to compare the numerical results from using our approach of the approximated DTBC, i.e. the sum-of-exponentials-ansatz (3) (with $\nu=2$ ) to the solution using the exact DTBC (2).

Example 2. As an example, we consider (1) on $0 \leq x \leq 1$ with $V_{-}=V_{+}=$ 0 , and initial data $\psi^{I}(x)=\exp \left(i 50 x-30(x-0.5)^{2}\right)$. The time evolution of the approximate solution $\left|\psi_{a}(x, t)\right|$ using the approximated DTBC with convolution coefficients $\left\{\tilde{s}_{n}\right\}$ and $L=10, L=20$, respectively, is shown in Fig. 3 (observe the viewing angle).

While one can observe some reflected wave when using the approximated DTBC with $L=10$, there are almost no reflections visible when using the approximated DTBC with $L=20$.

The goal is to investigate the long-time stability behaviour of the approximated DTBC with the sum-of-exponentials ansatz. The reference solution $\psi_{\text {ref }}$ with $\Delta x=1 / 400, \Delta t=2 \cdot 10^{-5}$ is obtained by using exact DTBCs $(2)$ at the ends $x=0$ and $x=1$. We vary the parameter $L=10,20,30,40$ in (3), find the corresponding approximate DTBCs, and show the relative error of the approximate solution, i.e. $\frac{\left\|\psi_{a}-\psi_{r e f}\right\|_{L_{2}}(t)}{\| \psi^{I}||_{L_{2}}}$. The result up to $n=15000$ 

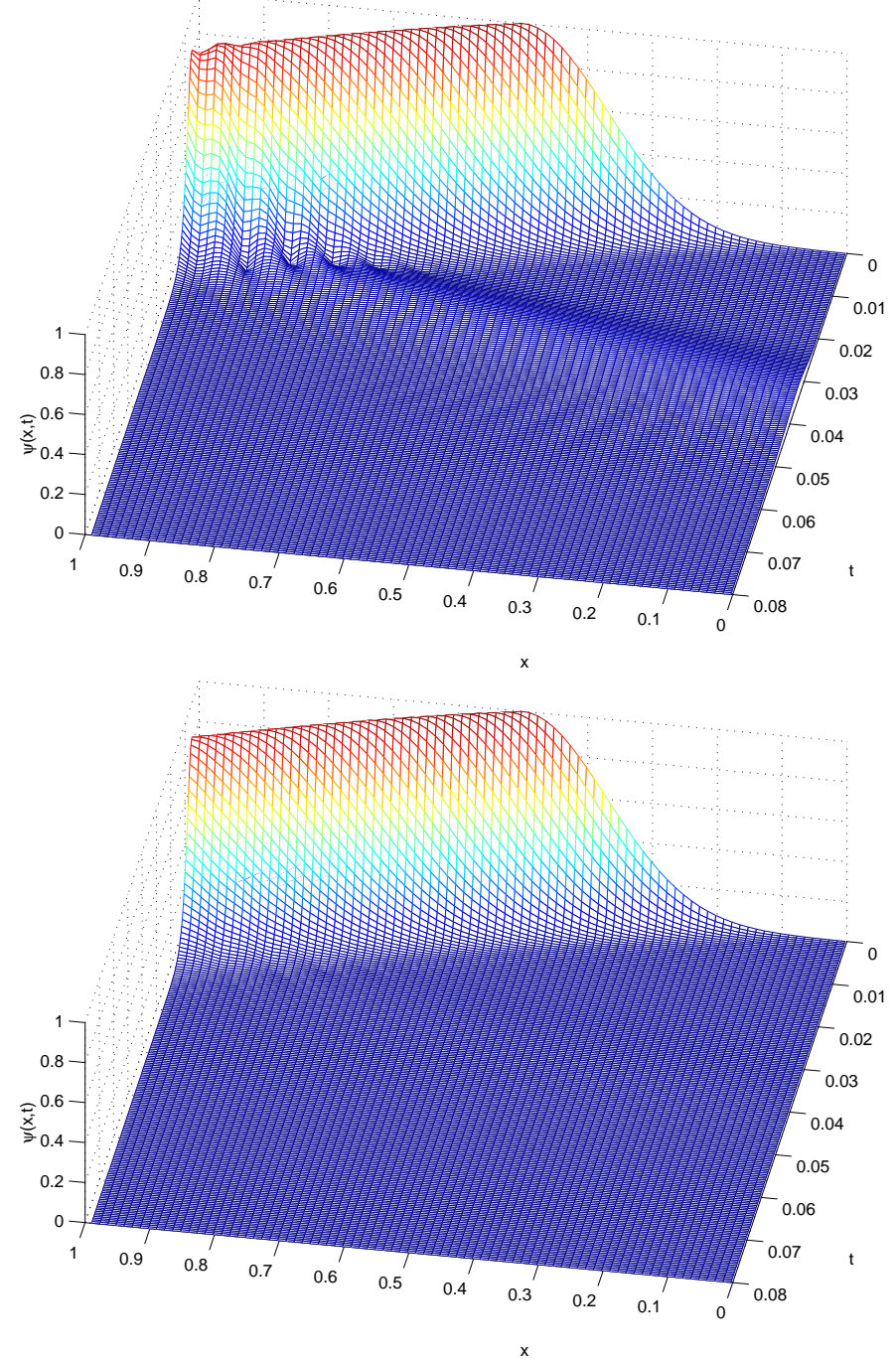

Fig. 3. Time evolution of $\left|\psi_{a}(x, t)\right|$ : The approximate convolution coefficients consisting of $L=10$ discrete exponentials give rise to a reflected wave (upper figure). Using $L=20$ discrete exponentials make reflections almost invisible (lower figure).

is shown in Fig. 4. Larger values of $L$ clearly yield more accurate coefficients and hence a more accurate solution $\psi_{a}$. Fig. 4 also shows the discretization error, i.e. $\frac{\left\|\psi_{r e f}-\psi_{a n}\right\|_{L_{2}}(t)}{\left\|\psi^{I}\right\|_{L_{2}}}$, where $\psi_{a n}$, the analytic solution of this example is explicitly computable.

Example 3. The second example considers (1) on $[0,2]$ with zero potential in the interior $(V(x) \equiv 0$ for $0<x<2)$ and $V(x) \equiv 4500$ outside the computa- 


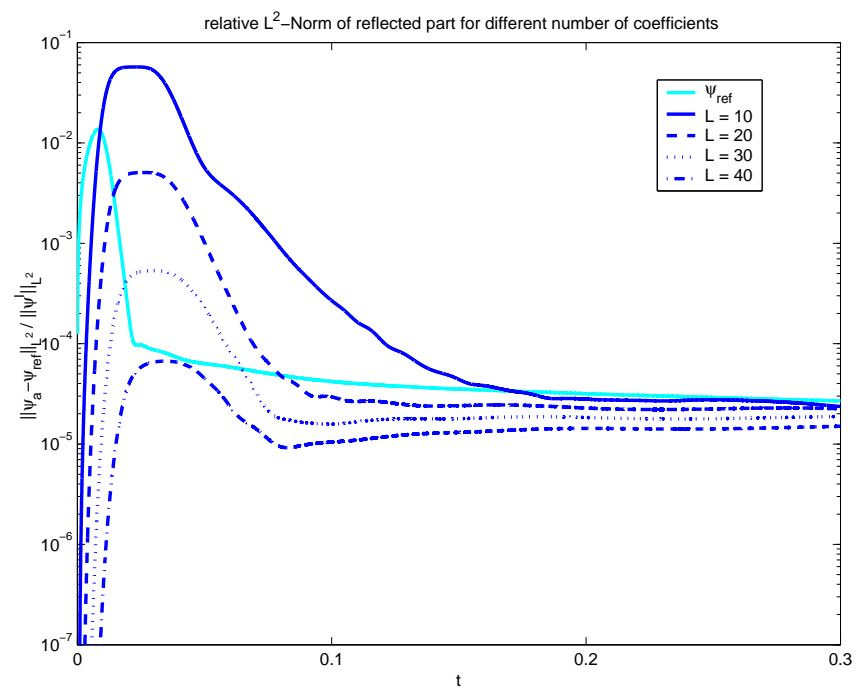

Fig. 4. Error of the approximate solution $\psi_{a}(t)$ with approximate convolution coefficients consisting of $L=10,20,30,40$ discrete exponentials and discretization error. $\psi_{\text {ref }}$ is the relative error of $\psi_{\text {ref }}$. The error-peak between $t=0.01$ and $t=0.02$ corresponds to the first reflected wave.

tional domain. The initial data is taken as $\left[\psi^{I}(x)=\exp \left(i 100 x-30(x-1)^{2}\right)\right]$, and this wave packet is partially reflected at the boundaries. We use the rather coarse space discretization $\Delta x=1 / 160$, the time step $\Delta t=2 \cdot 10^{-5}$, and the exact DTBC (2). The value of the potential is chosen such that at time $t=0.08$, i.e. after 4000 time steps $75 \%$ of the mass $\left(\|\psi(., t)\|_{2}^{2}\right)$ has left the domain. Fig. 5 shows the time decay of the discrete $\ell^{2}$-norm $\|\psi(., t)\|_{2}$ and the temporal evolution of the error $\left\|e_{L}(., t)\right\|_{2}:=\left\|\psi_{a}(., t)-\psi_{\text {ref }}(., t)\right\|_{2} /\left\|\psi^{I}\right\|_{L_{2}}$ when using an approximated DTBC with $L=20,30,40$. Additionally, we calculated for $L=20$ the coefficients $\left\{b_{l}, q_{l}\right\}$ for the "normalized parameters" $\Delta x=1, \Delta t=1, V=0$ and then used the Transformation rule 3.1 to calculate the coefficients $\left\{b_{l}^{*}, q_{l}^{*}\right\}$ for the desired parameters.

\section{Conclusion}

For numerical simulations of the Schrödinger equation one has to introduce artificial (preferable transparent) boundary conditions in order to confine the calculation to a finite region. Such TBCs are non-local in time (of convolution form). Hence, the numerical costs (just) for evaluating these BCs grow quadratically in time. And for long-time calculations it can easily outweigh the costs for solving the PDE inside the computational domain.

Here, we presented an efficient method to overcome this problem. We construct approximate DTBCs that are of a sum-of-exponential form and hence 


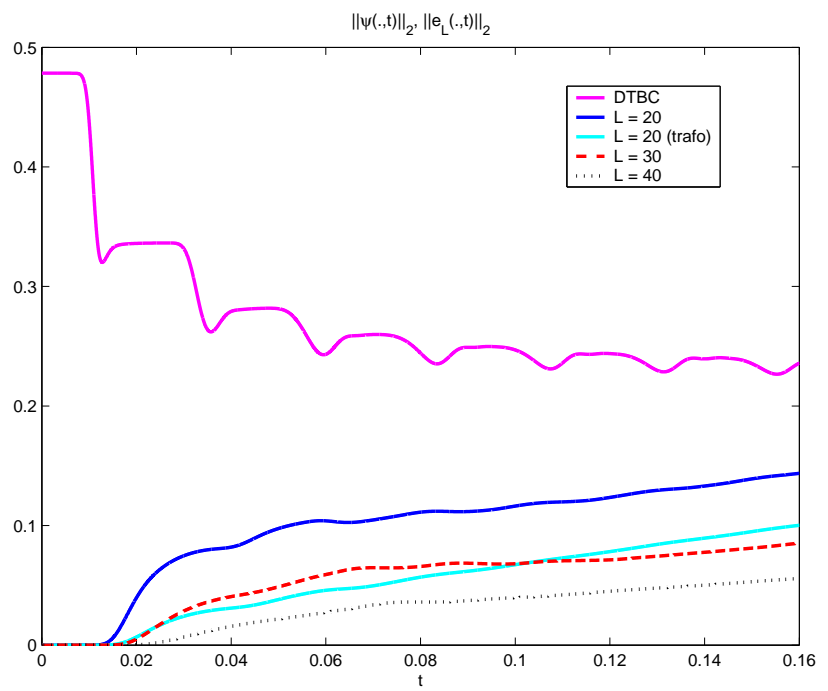

Fig. 5. Time evolution within the potential well $(V=4500)$ of $\|\psi(., t)\|_{2}$ and of the errors $\left\|e_{L}(., t)\right\|_{2}$ that are due to approximated DTBCs with $L=20,30,40$. " $L=20$ (trafo)" uses coefficients calculated by the Transformation rule 3.1.

only involve a linearly growing numerical effort. Moreover, these BCs yield very accurate solutions, and it was shown in Arnold et al. [2003] that the resulting initial-boundary value scheme is conditionally $\ell^{2}$-stable on $[0, T]$ as $\Delta t \rightarrow 0$ (e.g. for $0<\Delta t<\Delta t_{0}$ and $\Delta x=\Delta x_{0}=$ const.).

Acknowledgement. The first two authors were partially supported by the IHPNetwork HPRN-CT-2002-00282 from the EU and the DFG under Grant-No. AR 277/3-1. The second author was supported by the DFG Research Center "Mathematics for key technologies" (FZT 86) in Berlin. The third author was partially supported by RFBR-Grant No. 01-01-00520 and by Saarland University.

\section{References}

A. Arnold. Numerically absorbing boundary conditions for quantum evolution equations. VLSI Design, 6:313-319, 1998.

A. Arnold, M. Ehrhardt, and I. Sofronov. Discrete transparent boundary conditions for the Schrödinger equation: Fast calculation, approximation, and stability. Comm. Math. Sci., 1:501-556, 2003.

M. Ehrhardt and A. Arnold. Discrete transparent boundary conditions for the Schrödinger equation. Riv. Mat. Univ. Parma, 6:57-108, 2001.

I. Sofronov. Artificial boundary conditions of absolute transparency for twoand threedimensional external time-dependent scattering problems. Euro. J. Appl. Math., 9:561-588, 1998. 